\title{
Local Observability of Systems on Time Scales
}

\author{
Zbigniew Bartosiewicz \\ Faculty of Computer Science, Bialystok University of Technology, Wiejska 45a, 15-351 Bialystok, Poland
}

Correspondence should be addressed to Zbigniew Bartosiewicz; z.bartosiewicz@pb.edu.pl

Received 9 August 2013; Revised 10 October 2013; Accepted 10 October 2013

Academic Editor: Delfim F. M. Torres

Copyright (c) 2013 Zbigniew Bartosiewicz. This is an open access article distributed under the Creative Commons Attribution License, which permits unrestricted use, distribution, and reproduction in any medium, provided the original work is properly cited.

Analytic systems on an arbitrary time-scale are studied. As particular cases they include continuous-time and discrete-time systems. Several local observability properties are considered. They are characterized in a unified way using the language of real analytic geometry, ideals of germs of analytic functions, and their real radicals. It is shown that some properties related to observability are preserved under various discretizations of continuous-time systems.

\section{Introduction}

Local observability for nonlinear systems is defined in various ways [1-4]. As most of these concepts are introduced in the general system-theoretic setting via indistinguishability relation, they mean the same for continuous- and discretetime systems. We show here that some of these concepts may be studied in a unified way in the framework of systems on time-scales. A time-scale is a model of time, which can be continuous, discrete, or even mixed. Calculus on timescale is a unification of ordinary differential calculus and the calculus of finite differences. Delta differential equations may be used to model continuous- and discrete-time systems. In the discrete case, time-scale may be nonhomogeneous. It may be applied to systems that are obtained by nonuniform sampling or nonuniform Euler discretization of continuoustime systems.

We concentrate on strong, weak, and robust local observability of analytic systems. We show that the results obtained in $[2,3,5]$ may be extended to systems on arbitrary timescales. This is due to the fact that all the properties are characterized with the aid of the observation algebra of the system, which may be introduced in a universal way on all time-scales. Since the observation algebra consists of real functions defined on the state space, we can use the common procedures to derive the criteria of local observability. As in $[2,3]$ we use the language of local analytic geometry and real algebra to characterize weak and robust local observability.
Ideals of germs of analytic functions and real radicals of these ideals are used to express the criteria.

As an application of time-scale approach to local observability we consider discretization of continuous-time systems. This means replacing the standard derivative by the delta derivative on an appropriate discrete time-scale. We show that some of the properties related to observability are preserved under this operation. We will allow arbitrary discretizations: the discrete time-scale will not have to be homogeneous. Such nonuniform discretizations behave in a better way in many computations.

In Appendices we provide necessary information on time-scale calculus, local analytic geometry, and real algebra.

\section{Preliminaries}

Let $\mathbb{T}$ be a time-scale. We will assume that $\mathbb{\mathbb { T }}$ is forward infinite; that is, for every $t_{0} \in \mathbb{T}$ there are infinitely many elements of $\mathbb{T}$ that are greater than $t_{0}$. This will allow us to compute delta derivatives of arbitrary order at $t_{0}$. Let us consider a control system with output

$$
\begin{gathered}
\Sigma: \begin{array}{c}
x^{\Delta}(t)=f(x(t), u(t)) \\
y(t)=h(x(t))
\end{array}
\end{gathered}
$$

where $t \in \mathbb{T}, x(t) \in \mathbb{R}^{n}, y(t) \in \mathbb{R}^{r}$, and $u(t) \in \Omega$-arbitrary set. For $\omega \in \Omega$, let $f_{\omega}$ be defined by $f_{\omega}(x):=f(x, \omega)$. We assume that the maps $h$ and $f_{\omega}$ for every $\omega \in \Omega$ are analytic and that controls $u$ are piecewise constant functions of time. 
If $\mathbb{T}=\mathbb{R}$, then (1) is the standard continuous-time system

$$
\begin{gathered}
\dot{x}(t)=f(x(t), u(t)), \\
y(t)=h(x(t)) .
\end{gathered}
$$

For $\mathbb{T}=\mathbb{Z}(1)$ takes the form

$$
\begin{gathered}
x(t+1)-x(t)=f(x(t), u(t)), \\
y(t)=h(x(t)) .
\end{gathered}
$$

This can be rewritten in a more standard shift form as

$$
\begin{gathered}
x(t+1)=f(x(t), u(t))+x(t)=: g(x(t), u(t)), \\
y(t)=h(x(t)) .
\end{gathered}
$$

As there is a simple passage from $f$ to $g$ and vice versa, all statements for (3) may be translated to statements for (4).

Remark 1. The equation $x(t+1)=g(x(t), u(t))$ may be studied on an arbitrary set $X$ or on an analytic manifold $M$, if analyticity of the system is essential. But then we cannot pass to form (3), as to do this we need a linear space structure. Thus, one can argue that (4) is more general than (3). However, we concentrate here on local analytic problems, for which $\mathbb{R}^{n}$ is general enough.

By $\gamma\left(t, t_{0}, x_{0}, u\right)$ we denote the solution of the equation $x^{\Delta}=f(x, u)$ corresponding to control $u$ and the initial condition $x\left(t_{0}\right)=x_{0}$ and evaluated at time $t$.

Let $x_{1}, x_{2} \in \mathbb{R}^{n}$ and $t_{0} \in \mathbb{T}$. Then $x_{1}$ and $x_{2}$ are called indistinguishable at time $t_{0}$ if

$$
h\left(\gamma\left(t, t_{0}, x_{1}, u\right)\right)=h\left(\gamma\left(t, t_{0}, x_{2}, u\right)\right)
$$

for every control $u$ defined on $\left[t_{0}, t_{1}\right)_{\mathbb{T}}$ for some $t_{1} \in \mathbb{T}$, and every $t \in\left[t_{0}, t_{1}\right]_{\mathbb{T}}$ for which both sides of the equation are defined. Otherwise $x_{1}$ and $x_{2}$ are distinguishable at time $t_{0}$.

The states $x_{1}$ and $x_{2}$ are called indistinguishable if they are indistinguishable at time $t_{0}$ for every $t_{0} \in \mathbb{T}$. Otherwise $x_{1}$ and $x_{2}$ are distinguishable. Thus, $x_{1}$ and $x_{2}$ are distinguishable if they are distinguishable at some time $t_{0}$.

Remark 2. If the time-scale is not homogeneous, indistinguishability at time $t_{0}$ may depend on $t_{0}$. Though the systems we consider have "constant coefficients," that is, the map $f$ does not depend on time, inhomogeneity of the time-scale results in the behavior found in time-variant systems. This may be observed even for linear systems (see [6]).

Let $C^{\omega}\left(\mathbb{R}^{n}\right)$ denote the algebra of all real analytic functions on $\mathbb{R}^{n}$ and let $g: \mathbb{R}^{n} \rightarrow \mathbb{R}^{n}$ be analytic. Let us fix $t_{0} \in \mathbb{R}^{n}$. In [7] the following operator

$$
\Gamma_{g}^{t_{0}}: C^{\omega}\left(\mathbb{R}^{n}\right) \longrightarrow C^{\omega}\left(\mathbb{R}^{n}\right)
$$

was introduced as

$$
\left(\Gamma_{g}^{t_{0}} \varphi\right)(x):=\int_{0}^{1} \varphi^{\prime}\left(x+s \mu\left(t_{0}\right) g(x)\right) d s \cdot g(x),
$$

where $\varphi \in C^{\omega}\left(\mathbb{R}^{n}\right)$ and $\varphi^{\prime}$ is the gradient of $\varphi$ (a row vector).
Example 3. Let $\varphi=x^{i}$ be the $i$ th coordinate function on $\mathbb{R}^{n}$. Then $\varphi^{\prime}=e_{i}$-the (row) vector of the standard basis of $\mathbb{R}^{n}$ with 1 at the $i$-th position. For any $t_{0} \in \mathbb{T}$ we have

$$
\left(\Gamma_{g}^{t_{0}} x^{i}\right)(x)=e_{i} g(x)=g_{i}(x) .
$$

Observe that if $\mu\left(t_{0}\right)>0$ then

$$
\left(\Gamma_{g}^{t_{0}} \varphi\right)(x)=\frac{1}{\mu\left(t_{0}\right)}\left(\varphi\left(x+\mu\left(t_{0}\right) g(x)\right)-\varphi(x)\right) \text {. }
$$

On the other hand for $\mu\left(t_{0}\right)=0$ we obtain $\left(\Gamma_{g}^{t_{0}} \varphi\right)(x)=$ $\varphi^{\prime}(x) g(x)$, which is the standard Lie derivative of the function $\varphi$ along the vector field $g$. In general, when operator $\Gamma_{g}^{t_{0}}$ does not depend on $t_{0}$, we will denote it by $\Gamma_{g}$. This happens, for instance, if the time-scale is homogeneous.

Let $\sigma_{g}^{t_{0}}: C^{\omega}\left(\mathbb{R}^{n}\right) \rightarrow C^{\omega}\left(\mathbb{R}^{n}\right)$ be another map related to the function $g$ and the time $t_{0}$ defined by

$$
\left(\sigma_{g}^{t_{0}} \varphi\right)(x):=\varphi\left(x+\mu\left(t_{0}\right) g(x)\right) .
$$

If $\mu\left(t_{0}\right)=0$, then $\sigma_{g}^{t_{0}}$ is the identity map. In general we have an obvious property

Proposition 4. The map $\sigma_{g}^{t_{0}}$ is an endomorphism of the algebra $C^{\omega}\left(\mathbb{R}^{n}\right)$.

For $\mu\left(t_{0}\right)>0$ the operators $\Gamma_{g}^{t_{0}}$ and $\sigma_{g}^{t_{0}}$ are related by the following equality:

$$
\sigma_{g}^{t_{0}} \varphi=\varphi+\mu\left(t_{0}\right) \Gamma_{g}^{t_{0}} \varphi
$$

We also have the following generalization of the Leibniz rule.

\section{Proposition 5.}

$$
\Gamma_{g}^{t_{0}}(\varphi \psi)=\left(\Gamma_{g}^{t_{0}} \varphi\right)\left(\sigma_{g}^{t_{0}} \psi\right)+\varphi\left(\Gamma_{g}^{t_{0}} \psi\right)
$$

This property means that $\Gamma_{g}^{t_{0}}$ is a skew derivation of the algebra $C^{\omega}\left(\mathbb{R}^{n}\right)$ with respect to $\sigma_{g}^{t_{0}}$ or, in other words, that $\Gamma_{g}^{t_{0}}$ is a $\sigma_{g}^{t_{0}}$-derivation of this algebra. For $\mu\left(t_{0}\right)=0$ it is an ordinary derivation.

\section{Local Observability}

Let $H^{t_{0}}$ be a subset of $C^{\omega}\left(\mathbb{R}^{n}\right)$ consisting of functions of the form

$$
\Gamma_{g_{k}}^{t_{0}} \ldots \Gamma_{g_{1}}^{t_{0}} h_{i}
$$

where $i=1, \ldots, p, k \geq 0$ and $g_{i}=f_{\omega_{i}}$ for some $\omega_{i} \in \Omega$ and $i=1, \ldots, k$. If $k=0$ then this function is just $h_{i}$. Let

$$
H=\bigcup_{t_{0} \in \mathbb{T}} H^{t_{0}}
$$

Proposition 6. (i) The states $x_{1}$ and $x_{2}$ are indistinguishable at time $t_{0}$ if and only if for every $\varphi \in H^{t_{0}}, \varphi\left(x_{1}\right)=\varphi\left(x_{2}\right)$.

(ii) The states $x_{1}$ and $x_{2}$ are indistinguishable if and only if for every $\varphi \in H, \varphi\left(x_{1}\right)=\varphi\left(x_{2}\right)$. 
Proof. The statement (ii) was shown in [1] for continuoustime systems $(\mathbb{T}=\mathbb{R})$. In this case (i) and (ii) mean the same since indistinguishability at some $t_{0}$ is equivalent to indistinguishability. For an arbitrary time-scale (ii) follows from (i). The statement (i) for an arbitrary time-scale was shown in [8]. Analyticity of the control system and the functions from $H$ is essential in the proof.

Remark 7. Proposition 6 allows us to use the same language of analytic functions on $\mathbb{R}^{n}$ to study different observability properties of analytic systems on arbitrary time-scales as long as these properties are defined via the indistinguishability relation. It also implies that indistinguishability is an equivalence relation. This is not true for smooth systems (see [9]) or for analytic partially defined systems (see [10], where a different definition was developed to preserve this property for partially defined systems).

Let $\mathscr{H}$ denote the subalgebra of $C^{\omega}\left(\mathbb{R}^{n}\right)$ generated by $H$. It will be called the observation algebra of the system $\Sigma$. The elements of $\mathscr{H}$ are obtained by substituting functions from $H$ into polynomials of several variables with real coefficients. In particular, all constant functions belong to $\mathscr{H}$.

From Proposition 6 we get the following.

Proposition 8. The states $x_{1}$ and $x_{2}$ are indistinguishable if and only if for every $\varphi \in \mathscr{H}, \varphi\left(x_{1}\right)=\varphi\left(x_{2}\right)$.

We say that $\Sigma$ is observable if any two distinct states are distinguishable.

From the definition and Proposition 8 we obtain the following characterization.

Proposition 9. $\Sigma$ is observable if and only if for any distinct $x_{1}, x_{2} \in \mathbb{R}^{n}$ there is $\varphi \in \mathscr{H}$ such that $\varphi\left(x_{1}\right) \neq \varphi\left(x_{2}\right)$.

The condition stated in Proposition 9 is difficult to check. This is one of the reasons that a weaker concept of local observability seems to be more interesting. There are many different concepts of local observability and one concept has often a few different names. For the first two concepts we follow the terminology used in [4].

We say that $\sum$ is weakly locally observable at $x_{0}\left(\operatorname{WLO}\left(x_{0}\right)\right)$ if there is a neighborhood $U$ of $x_{0}$ such that for every $x \in U$, $x$ and $x_{0}$ are distinguishable.

Remark 10. Weak local observability at $x_{0}$ is in fact a weak property. It holds, for example, for the system

$$
x^{\Delta}=0, \quad y=x_{1}^{2}+\cdots+x_{n}^{2},
$$

at $x_{0}=0 \in \mathbb{R}^{n}$. Since all solutions of $x^{\Delta}=0$ are constant, time does not influence indistinguishability relation. To distinguish points we have to use only the output function. Clearly, it takes different values at 0 and any other point, so we can distinguish $x_{0}$ from any of its neighbors. Observe that local observability fails at any $x_{0} \neq 0$, if $n \geq 2$.

We say that $\Sigma$ is strongly locally observable at $x_{0}\left(\operatorname{SLO}\left(x_{0}\right)\right)$ if there is a neighborhood $U$ of $x_{0}$ such that for every distinct $x_{1}, x_{2} \in U, x_{1}$ and $x_{2}$ are distinguishable.
We say that $\Sigma$ is robustly locally observable at $x_{0}\left(\operatorname{RLO}\left(x_{0}\right)\right)$ if there is a neighborhood $U$ of $x_{0}$ such that $\Sigma$ is weakly locally observable at $x$ for every $x \in U$.

Robust local observability was introduced in [3] for continuous-time systems under the name "stable local observability." It means that the weak local observability at $x_{0}$ is stable or robust with respect to small perturbations of the initial condition $x_{0}$.

We call $\Sigma$ weakly locally observable (WLO) (strongly locally observable (SLO), and robustly locally observable (RLO), resp.), when it is weakly locally observable (strongly locally observable and robustly locally observable, resp.) at every $x \in \mathbb{R}^{n}$.

Let $d \mathscr{H}\left(x_{0}\right)$ denote the linear space of the differentials of functions from $\mathscr{H}$ taken at $x_{0}$. The following theorem is a simple extension of the result from [1].

Theorem 11. (a) If $\operatorname{dim} d \mathscr{H}\left(x_{0}\right)=n$, then $\Sigma$ is $\operatorname{SLO}\left(x_{0}\right)$.

(b) (for all $\left.x_{0} \in \mathbb{R}^{n}: \operatorname{dim} d \mathscr{H}\left(x_{0}\right)=n\right) \Rightarrow$ (for all $x_{0} \in \mathbb{R}^{n}: \Sigma$ is $\left.\operatorname{SLO}\left(x_{0}\right)\right) \Rightarrow(\exists X-a$ real analytic set in $\mathbb{R}^{n}:$ for all $\left.x_{0} \notin X: \operatorname{dim} d \mathscr{H}\left(x_{0}\right)=n\right)$.

Let us denote the condition $\operatorname{dim} d \mathscr{H}\left(x_{0}\right)=n$ by $\operatorname{HK}\left(x_{0}\right)$ (Hermann-Krener condition at $x_{0}$ ). The second part of Theorem 11 says that if we are interested in strong local observability at large, that is, at each point, then condition $\mathrm{HK}\left(x_{0}\right)$ is satisfied almost everywhere, so the gap between sufficient condition and necessary condition for strong local observability at large is quite narrow. However, when one is interested in local observability at a particular point of the state space, the Hermann-Krener condition may be far from being necessary (see [2]).

We have a nice gradation of different local observability concepts.

Proposition 12. $H K\left(x_{0}\right) \Rightarrow \sum$ is $S L O\left(x_{0}\right) \Rightarrow \sum$ is $R L O\left(x_{0}\right) \Rightarrow$ $\sum$ is $W L O\left(x_{0}\right)$.

None of the implications in Proposition 12 may be, in general, reversed.

Example 13. (a) Let $x \in \mathbb{R}, x^{\Delta}=0$, and $y=x^{3}$. Thus, $H=$ $\left\{x^{3}\right\}$. The system is observable, so it is also strongly locally observable at any $x$. But the Hermann-Krener condition fails at $x=0$.

(b) Let $x \in \mathbb{R}, x^{\Delta}=0$, and $y=x^{2}$. Then $H=\left\{x^{2}\right\}$. The system is robustly locally observable at 0 , but it is not strongly locally observable at this point.

(c) Let $x \in \mathbb{R}^{2}, x^{\Delta}=0$, and $y=x_{1}^{2}+x_{2}^{2}$. The system is weakly locally observable at 0 , but it is not robustly locally observable at this point.

But we have an important, though obvious, global equivalence.

Proposition 14. $\Sigma$ is $R L O\left(x_{0}\right)$ for every $x_{0} \in \mathbb{R}^{n}$ if and only if $\Sigma$ is $W L O\left(x_{0}\right)$ for every $x_{0} \in \mathbb{R}^{n}$. In other words, $\Sigma$ is $R L O$ if and only if $\Sigma$ is $W L O$.

By $\mathcal{O}_{x}$ we will denote the algebra of germs at $x$ of analytic functions on $\mathbb{R}^{n}$ (see Appendix B). Let $J_{x}$ be the ideal of $\mathcal{O}_{x}$ generated by germs at $x$ of functions from $\mathscr{H}$ that vanish at $x$. 
For $x \in \mathbb{R}^{n}$ and for an ideal $J$ of $\mathcal{O}_{x}$, let $Z(J)$ be the germ at $x$ of the zero-set of $J$. Since $J$ is finitely generated $\left(\mathcal{O}_{x}\right.$ is Noetherian), $Z(J)$ is well defined. Let $I(Z(J))$ be the ideal of $\mathcal{O}_{x}$ consisting of all germs of analytic functions that vanish on $Z(J)$.

Lemma 15. The following conditions are equivalent:

(a) $Z\left(J_{x_{0}}\right) \neq\left\{x_{0}\right\}$;

(b) arbitrarily close to $x_{0}$ there is $x$ such that $\varphi\left(x_{0}\right)=\varphi(x)$ for every $\varphi \in \mathscr{H}$.

Proof. (a) holds if and only if arbitrarily close to $x_{0}$ there is $x$ such that all representatives of germs $\varphi \in J_{x_{0}}$ (all defined in some neighborhood of $x_{0}$ ) are 0 at $x$. This is equivalent to the fact that all functions $\varphi \in \mathscr{H}$ take on the same values at $x_{0}$ and this $x$, which means precisely (b).

To characterize weak local observability we use the concept of real radical (see Appendix B).

\section{Theorem 16.}

(a) $\Sigma$ is $W L O\left(x_{0}\right)$ if and only if $\sqrt[R]{J_{x_{0}}}=m_{x_{0}}$.

(b) $H K\left(x_{0}\right)$ if and only if $J_{x_{0}}=m_{x_{0}}$.

Proof. (a) $\Sigma$ is not weakly locally observable at $x_{0}$ if and only if arbitrarily close to $x_{0}$ there is $x$ such that $x_{0}$ and $x$ are indistinguishable. By Lemma 15, the last statement is equivalent to the condition $Z\left(J_{x_{0}}\right) \neq\left\{x_{0}\right\}$, which in turn means that $I\left(Z\left(J_{x_{0}}\right)\right) \neq I\left(\left\{x_{0}\right\}\right)$. But $I\left(\left\{x_{0}\right\}\right)=m_{x_{0}}$, so from Theorem B.1 the last inequality is equivalent to the condition $\sqrt[R]{J_{x_{0}}} \neq m_{x_{0}}$. This gives the equivalence of both sides in (a).

(b) It is enough to prove the proposition for $x=0$ in $\mathbb{R}^{n}$.

Observe that $d \mathscr{H}(x)=d J_{x}(x)$ for every $x$. Thus, if $J_{0}=m_{0}$, then $d \mathscr{H}(0)$ contains all the differentials $d x_{i}$ for $i=1, \ldots, n$, which are linearly independent.

On the other hand, the condition $\operatorname{dim} d \mathscr{H}(0)=n$ implies that in a neighborhood $U$ of 0 , there are functions $\varphi_{1}, \ldots, \varphi_{n}$ whose germs belong to $J_{0}$ and the differentials at $0, d \varphi_{i}(0)$ are linearly independent. We may write $\varphi_{i}=\sum_{j} \Phi_{i j} x_{j}$ for some analytic functions $\Phi_{i j}$ on $U$ (sufficiently small). Then $d \varphi_{i}(0)=\sum_{j} \Phi_{i j}(0) d x_{j}$. This means that the matrix $\Phi=$ $\left(\Phi_{i j}\right)$ is invertible at 0 and then in some neighborhood of 0. Let $\Psi=\Phi^{-1}$. Then the germs of elements of $\Psi$ are in $\mathcal{O}_{x}$ and $d x_{i}=\sum_{j} \Psi_{i j} d \varphi_{j}$ which means that $\varphi_{1}, \ldots, \varphi_{n}$ generate $m_{0}$.

Let $G$ be a subset of $\mathcal{O}_{x}$. By $D(G)$ we will denote the ideal of $\mathcal{O}_{x}$ generated by Jacobians $\partial\left(\varphi_{1}, \ldots, \varphi_{n}\right) / \partial\left(x_{1}, \ldots, x_{n}\right)$, where $\varphi_{i} \in G$. Observe that these Jacobians are well defined on germs of functions. If $\mathscr{G}$ is a family of real analytic functions on an open set $U$ in $\mathbb{R}^{n}$, then similarly we define the Jacobian ideal $D(\mathscr{G})$ in $\mathcal{O}_{U}$. Furthermore, there is a simple relation between Jacobian ideals for functions and germs of functions. If $\mathscr{G}$ is a family of analytic functions on $U$, and $x_{0} \in U$ then we have

$$
D(\mathscr{G})_{x_{0}}=D\left(\mathscr{G}_{x_{0}}\right) .
$$

Now, for a point $x \in \mathbb{R}^{n}$, we define a sequence of ideals in $\mathcal{O}_{x}$ related to the system $\Sigma$. Let $I_{x}^{(0)}:=(0)$ and $I_{x}^{(k+1)}:=$ $\sqrt[R]{D\left(\mathscr{H}_{x} \cup I_{x}^{(k)}\right)}$. It is clear that instead of $\mathscr{H}_{x}$ in this definition one can take the previously defined ideal $J_{x}$; that is, $I_{x}^{(k+1)}=$ $\sqrt[\mathbb{R}]{D\left(J_{x} \cup I_{x}^{(k)}\right)}$. This leads to the following generalization of statement (b) of Theorem 16.

Corollary 17. $H K\left(x_{0}\right) \Leftrightarrow J_{x_{0}}=m_{x_{0}} \Leftrightarrow I_{x_{0}}^{(1)}=\mathcal{O}_{x_{0}}$.

The ideals $I_{x}^{(k)}$ will be the main tools in studying robust local observability. First we prove the basic fact.

Proposition 18. For any $k \geq 0, I_{x}^{(k)} \subseteq I_{x}^{(k+1)}$, and there is $s \geq 0$ such that $I_{x}^{(s)}=I_{x}^{(s+1)}$.

Proof. To prove the first part we proceed by induction. It is clear that $I_{x}^{(0)} \subseteq I_{x}^{(1)}$, so assume that $I_{x}^{(k)} \subseteq I_{x}^{(k+1)}$ for some $k \geq 0$. Then also $\mathscr{H}_{x} \cup I_{x}^{(k)} \subseteq \mathscr{H}_{x} \cup I_{x}^{(k+1)}$ and $D\left(\mathscr{H}_{x} \cup I_{x}^{(k)}\right) \subseteq D\left(\mathscr{H}_{x} \cup I_{x}^{(k+1)}\right)$, so finally $\sqrt[R]{D\left(\mathscr{H}_{x} \cup I_{x}^{(k)}\right)} \subseteq$ $\sqrt[\mathbb{R}]{D\left(\mathscr{H}_{x} \cup I_{x}^{(k+1)}\right)}$. This means that $I_{x}^{(k+1)} \subseteq I_{x}^{(k+2)}$. Since the ring $\mathcal{O}_{x}$ is Noetherian the sequence of ideals $I_{x}^{(k)}$ must stabilize at some $s$.

Now we can characterize robust local observability.

Theorem 19. System $\Sigma$ is $R L O\left(x_{0}\right)$ if and only if $I_{x_{0}}^{(s)}=\mathcal{O}_{x_{0}}$ for some $s>0$.

The proof of Theorem 19 will rely on several lemmas. They appeared in a similar form in [3]. However, there were a few flaws in the proofs, which are now corrected.

Let $\mathscr{G}$ be a family of analytic functions on some open set $U \subset \mathbb{R}^{n}$. Denote by $S_{x}(\mathscr{G})$ the germ at $x$ of the level set of $\mathscr{G}$ that passes through $x$. Thus

$$
S_{x}(\mathscr{G})=\{y \in U: \varphi(x)=\varphi(y) \text { for } \varphi \in \mathscr{G}\}_{x} .
$$

The set-germ $S_{x}(\mathscr{G})$ is a germ of analytic set. One of the representatives of $S_{x}(\mathscr{G})$ is the analytic set in $U:\{y \in U$ : $\varphi(y)=\varphi(x)$ for $\varphi \in \mathscr{G}\}$.

Lemma 20. Let $U$ be an open subset of $\mathbb{R}^{n}$ and let $X$ be an analytic set in $U$. Consider a family $\mathscr{G}$ of analytic functions on $U$. If for every $x \notin X: S_{x}(\mathscr{G})=\{x\}$, then for every $x \in X$ : $S_{x}(\mathscr{G}) \subset X_{x}$.

Proof. Suppose that there is $x \in X$ such that $S_{x}(\mathscr{G}) \not \subset X_{x}$. This means that for every representatives $\widetilde{S}_{x}(\mathscr{G})$ and $\widetilde{X}_{x}$ we have $\widetilde{S}_{x}(\mathscr{G}) \not \subset \widetilde{X}_{x}$. Take $\widetilde{X}_{x}:=X$ and arbitrarily small neighborhood $V$ of $x$ in $U$. Let $\widetilde{S}_{x}(\mathscr{G})$ be a representative of $S_{x}(\mathscr{G})$ in $V$. Then there is $y \in \widetilde{S}_{x}(\mathscr{G})$ such that $y \notin X$. Take a sequence $\left(y_{n}\right)$ of such points converging to $x$. We may assume that all these points belong to some (large enough) representative $Y$ of $S_{x}(\mathscr{G})$ and that $Y$ is an analytic set. Only finite number of points $y_{n}$ may be isolated points of $Y$. This means that arbitrarily close to $x$ there is a point $y_{n}$ for which $S_{y_{n}}(\mathscr{G}) \neq\left\{y_{n}\right\}$. Thus we get a contradiction. 
Lemma 21. Let $U$ be an open subset of $\mathbb{R}^{n}$ and let $\mathscr{G}$ be a family of analytic functions on $U$. For every $x \in U$ : if $x \notin Z(D(\mathscr{G}))$, then $S_{x}(\mathscr{G})=\{x\}$.

Proof. If $x \notin Z(D(\mathscr{G}))$, then there are functions $\varphi_{1}, \ldots, \varphi_{n} \in$ $G$ such that

$$
\frac{\partial\left(\varphi_{1}, \ldots, \varphi_{n}\right)}{\partial\left(x_{1}, \ldots, x_{n}\right)}(x) \neq 0 .
$$

Thus, the map $\bar{x} \mapsto\left(\varphi_{1}(\bar{x}), \ldots, \varphi_{n}(\bar{x})\right)$ is injective in a neighborhood of $x$. This implies that $S_{x}(\mathscr{G})=\{x\}$.

Lemma 22. Assume that $X_{x_{0}}^{(k)} \neq X_{x_{0}}^{(k+1)}$ for some $k \geq 0$. Then there is a neighborhood $U$ of $x_{0}$ in $\mathbb{R}^{n}$ and a representative $\widetilde{X}_{x_{0}}^{(k+1)}$ of $X_{x_{0}}^{(k+1)}$ in $U$ such that for every $x \in U$ :

$$
x \notin \widetilde{X}_{x_{0}}^{(k+1)} \Longrightarrow S_{x}(\mathscr{H})=\{x\} .
$$

Proof. First observe that $Z\left(D\left(\mathscr{H}_{\mid U}\right)\right)$ is a representative of $Z\left(D\left(\mathscr{H}_{x_{0}}\right)\right)$. We proceed by induction. Let $k=0$ and assume that $X_{x_{0}}^{(1)} \neq X_{x_{0}}^{(0)}=\mathbb{R}_{x_{0}}^{n}$. Take any neighborhood $U$ of $x_{0}$. Since $X_{x_{0}}^{(1)}=Z\left(D\left(\mathscr{H}_{x_{0}}\right)\right)$, then $Z\left(D\left(\mathscr{H}_{\mid U}\right)\right)=: \widetilde{X}_{x_{0}}^{(1)}$ is a representative of $X_{x_{0}}^{(1)}$ in $U$. If $x \notin \widetilde{X}_{x_{0}}^{(1)}$, then by Lemma 21, $S_{x}(\mathscr{H})=S_{x}\left(\mathscr{H}_{\mid U}\right)=\{x\}$.

Now assume that the statement of the lemma holds for $k-1 \geq 0$. Hence, there is a neighborhood $U$ of $x_{0}$ and a representative $\widetilde{X}_{x_{0}}^{(k)}=Z\left(\varphi_{1}, \ldots, \varphi_{s}\right)$ of $X_{x_{0}}^{(k)}$ such that if $x \in U$ and $x \notin \widetilde{X}_{x_{0}}^{(k)}$, then $S_{x}(\mathscr{H})=\{x\}$. The functions $\varphi_{1}, \ldots, \varphi_{s}$ are representatives on $U$ of generators of $I_{x_{0}}^{(k)}$. The set $\widetilde{X}_{x_{0}}^{(k+1)}:=Z\left(D\left(\mathscr{H}_{\mid U} \cup\left\{\varphi_{1}, \ldots, \varphi_{s}\right\}\right)\right)$ is a representative of $X_{x_{0}}^{(k+1)}$. Clearly $\widetilde{X}_{x_{0}}^{(k+1)} \subseteq \widetilde{X}_{x_{0}}^{(k)}$. Take $x \in U$ such that $x \notin \widetilde{X}_{x_{0}}^{(k+1)}$. If $x \notin \widetilde{X}_{x_{0}}^{(k)}$, then $S_{x}(\mathscr{H})=\{x\}$. Assume then that $x \in \widetilde{X}_{x_{0}}^{(k)}$. From Lemma 20 we get $S_{x}(\mathscr{H}) \subseteq\left(\widetilde{X}_{x_{0}}^{(k)}\right)_{x}$. Then $S_{x}(\mathscr{H})=S_{x}(\mathscr{H}) \cap\left(\widetilde{X}_{x_{0}}^{(k)}\right)_{x}=S_{x}\left(\mathscr{H}_{\mid U} \cup\left\{\varphi_{1}, \ldots, \varphi_{s}\right\}\right)$. Since $x \notin Z\left(D\left(\mathscr{H}_{\mid U} \cup\left\{\varphi_{1}, \ldots, \varphi_{s}\right\}\right)\right)$, then, by Lemma 21, $S_{x}(\mathscr{H})=S_{x}\left(\mathscr{H}_{\mid U} \cup\left\{\varphi_{1}, \ldots, \varphi_{s}\right\}\right)=\{x\}$. This finishes the inductive step of the proof.

Lemma 23. Let $x_{0} \in U \subset \mathbb{R}^{n}$, where $U$ is open, and let $\mathscr{G}$ be a family of analytic functions on $U$. If $D(\mathscr{G})=0$ (zero ideal), then arbitrarily close to $x_{0}$ there is $x \in U$ such that $S_{x}(\mathscr{G}) \neq\{x\}$.

Proof. Let

$$
s=\max _{\substack{x \in U \\ \varphi_{i} \in \mathscr{G}}} \operatorname{rank} \frac{\partial\left(\varphi_{1}, \ldots, \varphi_{n}\right)}{\partial\left(x_{1}, \ldots, x_{n}\right)}(x) .
$$

Then $s<n$ and arbitrarily close to $x_{0}$ there is $x \in$ $U$ and $\varphi_{1}, \ldots, \varphi_{n} \in \mathscr{G}$ such that $\operatorname{rank}\left(\partial\left(\varphi_{1}, \ldots, \varphi_{n}\right) /\right.$ $\left.\partial\left(x_{1}, \ldots, x_{n}\right)\right)(x)=s$. This rank is preserved in some neighborhood $V$ of $x$. Thus, we may assume that gradients of $\varphi_{1}, \ldots, \varphi_{s}$ are linearly independent at every point of $V$ and span $d \mathscr{G}(\bar{x})$ for $\bar{x} \in V$. Then by Frobenius Theorem, $V$ is a union of integral manifolds of codistribution $d \mathscr{G}$. The integral manifolds are the level sets of $\mathscr{G}$ and have dimension greater than or equals to 1 . This means that $S_{x}(\mathscr{G}) \neq\{x\}$.
Lemma 24. Let $U$ be an open subset of $\mathbb{R}^{n}$ and let $\varphi_{1}, \ldots, \varphi_{k}$ be analytic functions on $U$ whose gradients are linearly independent at each point of $U$. Let $Y=Z\left(\varphi_{1}, \ldots, \varphi_{k}\right), x_{0} \in Y$, and let $\mathscr{G}$ be a family of analytic functions on $U$.

If $Y \subset Z\left(D\left(\mathscr{G} \cup\left\{\varphi_{1}, \ldots, \varphi_{k}\right\}\right)\right)$, then arbitrarily close to $x_{0}$ there is $x \in Y$ such that $S_{x}(\mathscr{G}) \neq\{x\}$.

Proof. Changing the coordinates we can obtain $\varphi_{i}=x_{i}, i=$ $1, \ldots, k$. Let $\psi_{1}, \ldots, \psi_{n-k} \in \mathscr{G}$. Then for $x \in Y$ we have

$$
\begin{aligned}
& 0=\operatorname{det}\left[\begin{array}{c}
\nabla \varphi_{1}(x) \\
\vdots \\
\nabla \varphi_{k}(x) \\
\nabla \psi_{1}(x) \\
\vdots \\
\nabla \psi_{n-k}(x)
\end{array}\right] \\
& =\operatorname{det}\left[\left(\frac{\partial \psi_{i}}{\partial x_{j}}(x)\right)_{\substack{i=1, \ldots, n-k \\
j=1, \ldots, n}}^{0}\right] \\
& =\operatorname{det}\left(\frac{\partial \psi_{i}(x)}{\partial x_{j}}\right)_{\substack{i=1, \ldots, n-k \\
j=k+1, \ldots, n}} \text {. }
\end{aligned}
$$

Note that $Y$ is an analytic manifold and the last term above is actually the Jacobian of a map defined on $Y$. Hence, after restricting to the manifold $Y$, we get $D\left(\mathscr{G}_{\mid Y}\right)=0$. From Lemma 23, arbitrarily close to $x_{0}$ there is $x \in Y$ such that $S_{x}\left(\mathscr{G}_{\mid Y}\right) \neq\{x\}$. But $S_{x}\left(\mathscr{G}_{\mid Y}\right) \subset S_{x}(\mathscr{G})$ so also $S_{x}(\mathscr{G}) \neq\{x\}$.

Lemma 25. Assume that $X_{x_{0}}^{(s)}=X_{x_{0}}^{(s+1)}$ for some $s \geq 0$ and $X_{x_{0}}^{(s)} \neq \emptyset$. Then in every neighborhood of $x_{0}$ there is $x$ such that $S_{x}(\mathscr{H}) \neq\{x\}$.

Proof. Let $\varphi_{1}, \ldots, \varphi_{k}$ be representatives of generators of the ideal $I_{x_{0}}^{(s)}$, defined on some common neighborhood $U$ of $x_{0}$. Then $\widetilde{X}_{x_{0}}^{(s)}:=Z\left(\varphi_{1}, \ldots, \varphi_{k}\right)$ is a representative of $X_{x_{0}}^{(s)}$ in $U$. In every neighborhood of $x_{0}$ one can find a regular point of the analytic set $\widetilde{X}_{x_{0}}^{(s)}($ see $[11,12])$. Let $\bar{x}$ be such a point and let $V$ be a neighborhood of $\bar{x}$ in $\mathbb{R}^{n}$ such that $Y:=V \cap \widetilde{X}_{x_{0}}^{(s)}$ is an analytic manifold. Then $Y=Z\left(\varphi_{1 \mid V}, \ldots, \varphi_{k \mid V}\right)$. We may assume that the gradients of $\varphi_{1 \mid V}, \ldots, \varphi_{k \mid V}$ are linearly independent on $Y$. Otherwise, after possible shrinking of $V$, we can remove the functions whose gradients are linear combinations of the gradients of other functions. Let $x \in Y$. Then $x \in \widetilde{X}_{x_{0}}^{(s+1)}:=Z\left(D\left(\mathscr{H}_{\mid U} \cup\left\{\varphi_{1}, \ldots, \varphi_{k}\right\}\right)\right)$ so that $Y \subset$ $Z\left(D\left(\mathscr{H}_{\mid V} \cup\left\{\varphi_{1 \mid V}, \ldots, \varphi_{k \mid V}\right\}\right)\right)$. The statement of the lemma follows now from Lemma 24.

Proof of Theorem 19. Sufficiency. Assume that $I_{x_{0}}^{(s)}=\mathcal{O}_{x_{0}}$ for some $s>0$. This means that $X_{x_{0}}^{(s)}=\emptyset$. From Lemma 22 it follows that nontrivial set-germs $S_{x}(\mathscr{H})$ (i.e., different from $\{x\})$ may be found only in $\widetilde{X}_{x_{0}}^{(s)}$-some representative of $X_{x_{0}}^{(s)}$. 
But $X_{x_{0}}^{(s)}=\emptyset$ so in some neighborhood of $x_{0}$ the level setgerms of $\mathscr{H}$ must be trivial. This means that $\Sigma$ is robustly locally observable at $x_{0}$.

Necessity. Assume that $\widetilde{X}_{x_{0}}^{(s)}=\widetilde{X}_{x_{0}}^{(s+1)}$ for some $s \geq 0$. From Lemma 25 it follows that $\sum$ is not robustly locally observable.

The statements of Corollary 17, Proposition 18, and Theorem 19 can be translated into the language of germs of analytic sets. Let $X_{x}^{(k)}=Z\left(I_{x}^{(k)}\right)$. Because the ideals $I_{x}^{(k)}$ are real, we also get $J\left(X_{x}^{(k)}\right)=I_{x}^{(k)}$, so there is one-to-one correspondence between the ideals and the set-germs. We have then the following.

Corollary 26. Let $x_{0} \in \mathbb{R}^{n}$.

(a) $H K\left(x_{0}\right)$ if and only if $X_{x_{0}}^{(1)}=\emptyset$.

(b) For every $k \geq 0, X_{x_{0}}^{(k)} \supseteq X_{x_{0}}^{(k+1)}$, and for some $s \geq 0$, $X_{x_{0}}^{(s)}=X_{x_{0}}^{(s+1)}$.

(c) $\sum$ is $R L O\left(x_{0}\right)$ if and only if for some $s \geq 0, X_{x_{0}}^{(s)}=\emptyset$.

Example 27. Consider the following system:

$$
\begin{gathered}
x_{1}^{\Delta}=x_{1} u, \\
x_{2}^{\Delta}=x_{2}, \\
x_{3}^{\Delta}=0, \\
y=x_{1}^{2}+x_{2}^{2}+x_{3}^{2},
\end{gathered}
$$

and choose $x_{0}=0$. Then for an arbitrary time-scale we get $\mathscr{H}=\mathbb{R}\left[x_{1}^{2}, x_{2}^{2}, x_{3}^{2}\right]$ and $I_{x_{0}}^{(1)}=J_{x_{0}}=\left(x_{1} x_{2} x_{3}\right)$. Thus $X_{x_{0}}^{(1)}$ is the germ of a union of three planes intersecting at 0 . In the next step we obtain $I_{x_{0}}^{(2)}=\sqrt[\mathbb{R}]{\left(x_{1}^{2} x_{2}^{2}, x_{2}^{2} x_{3}^{2}, x_{1}^{2} x_{3}^{2}\right)}=$ $\left(x_{1} x_{2}, x_{2} x_{3}, x_{1} x_{3}\right)$. A quick calculation shows that $X_{x_{0}}^{(2)}$ is the germ of a union of three lines intersecting at 0 . Finally, $I_{x_{0}}^{(3)}=$ $m_{x_{0}}$ and then $I_{x_{0}}^{(4)}=\mathcal{O}_{x_{0}}$, so $\Sigma$ is robustly locally observable at $x_{0}$.

\section{Discretization}

As we are going to consider several time-scales, we will denote the graininess function on the time-scale $\mathbb{T}$ by $\mu_{\mathbb{T}}$. A timescale $\mathbb{T}$ is called discrete, if $\mu_{\mathbb{T}}(t)>0$ for all $t \in \mathbb{T}$.

Let $\Sigma$ be the continuous-time system

$$
\dot{x}=f(x, u), \quad y=h(x)
$$

and let $\Sigma_{\mathbb{T}}$ be its discretization

$$
x^{\Delta}=f(x, u), \quad y=h(x)
$$

on a discrete time-scale $\mathbb{T}$. Usually $\mathbb{T}$ is equal to $c \mathbb{Z}$ for some $c>0$, but nonhomogeneous time-scales are allowed as well.
In the discretized system the ordinary derivative is replaced by the delta derivative on the discrete time-scale.

Thus, (23) is replaced with

$$
\frac{x\left(t+\mu_{\mathbb{T}}(t)\right)-x(t)}{\mu_{\mathbb{T}}(t)}=f(x(t), u(t)), \quad y(t)=h(x(t))
$$

for $t \in \mathbb{T}$.

Let $\mathscr{H}$ denote the observation algebra of the system $\Sigma$ and $\mathscr{H}_{\mathbb{T}}$ the observation algebra of the system $\Sigma_{\mathbb{T}}$. Observe that each generator of $\mathscr{H}$ may be approximated by a corresponding generator of $\mathscr{H}_{\pi}$ for $\mu(t)$ sufficiently small. This follows from the form of the operators $\Gamma_{g}^{t_{0}}$ on $\mathbb{R}$ and $\mathbb{T}$, which is used in this procedure.

A natural question is which properties related to observability are preserved under discretization.

Proposition 28. If $x_{1}$ and $x_{2}$ are distinguishable by $\Sigma$, then there is $\widehat{\mu}>0$ such that $x_{1}$ and $x_{2}$ are distinguishable by $\Sigma_{\mathbb{T}}$ at $t \in \mathbb{T}$ whenever $\mu_{\mathbb{T}}(t)<\widehat{\mu}$.

Proof. Suppose that for every $\widehat{\mu}>0$ there are a time-scale $\mathbb{T}$ and $t \in \mathbb{T}$ with $0<\mu_{\mathbb{T}}(t)<\widehat{\mu}$ such that $x_{1}$ and $x_{2}$ are indistinguishable by $\Sigma_{\mathbb{T}}$ at $t$. This means that that there is a sequence $\left(\mathbb{T}_{i}\right)$ of time-scales and a sequence $\left(t_{i}\right)$ of real numbers such that $t_{i} \in \mathbb{T}_{i}$ and $\mu_{\mathbb{T}_{i}}\left(t_{i}\right) \rightarrow 0$ when $i \rightarrow \infty$ and for every $\varphi \in \mathscr{H}_{\mathbb{T}_{i}}^{t_{i}}, \varphi\left(x_{1}\right)=\varphi\left(x_{2}\right)$. Every function $\psi \in \mathscr{H}$ may be approximated by functions from $\mathscr{H}_{\mathbb{T}_{i}}^{t_{i}}$; that is, there is a sequence of functions $\left(\phi_{i}\right)$ such that $\varphi_{i} \in \mathscr{H}_{\mathbb{T}_{i}}^{t_{i}}$ and $\varphi_{i} \rightarrow \psi$ on some compact set containing $x_{1}$ and $x_{2}$. This implies that $\psi\left(x_{1}\right)=\psi\left(x_{2}\right)$, so $x_{1}$ and $x_{2}$ are indistinguishable by $\Sigma$.

In particular, distinguishability of $x_{1}$ and $x_{2}$ is preserved for quantum discretization, where $\mathbb{T}=q^{\mathbb{N}}$, if $q$ is sufficiently close to 1.

We will show now that the Hermann-Krener rank condition is preserved under discretization. Let $\mathrm{HK}\left(x_{0}\right)$ denote the Hermann-Krener condition at $x_{0}$ for the system $\Sigma$ and $\mathrm{HK}_{\mathbb{T}}\left(x_{0}\right)$ for the system $\Sigma_{\mathbb{T}}$.

Proposition 29. If $H K\left(x_{0}\right)$, then there is $\widehat{\mu}>0$ such that for every time-scale $\mathbb{\mathbb { V }}$ if there is $t \in \mathbb{W}$ with $0<\mu_{\mathbb{V}}(t)<\widehat{\mu}$, then $H K_{\mathbb{T}}\left(x_{0}\right)$.

Proof. Assume that $\mathrm{HK}\left(x_{0}\right)$ holds. Then there are $\psi_{1}, \ldots, \psi_{n} \in \mathscr{H}$ such that $d \psi_{1}\left(x_{0}\right), \ldots, d \psi_{n}\left(x_{0}\right)$ are linearly independent. Each $\psi_{i}$ may be approximated by some $\varphi_{i} \in \mathscr{H}_{\mathbb{T}}^{t}$ for $t \in \mathbb{T}$ and $\mu_{\mathbb{T}}(t)$ sufficiently small. Observe that the functions $\varphi_{i}$ depend actually on the parameter $\mu_{\mathbb{T}}(t)$ and this dependence is continuous. For $\mu_{\mathbb{}}(t)$ sufficiently small also $d \varphi_{1}\left(x_{0}\right), \ldots, d \varphi_{n}\left(x_{0}\right)$ will be linearly independent. This means that $\mathrm{HK}_{\mathbb{T}}\left(x_{0}\right)$ holds for such $\mathbb{T}$.

The converse of Proposition 29 does not hold.

Example 30. Let $\Sigma$ be

$$
\dot{x}_{1}=-u, \quad \dot{x}_{2}=3 x_{1}^{2} u, \quad y=x_{1}^{3}+x_{2} .
$$


The observation algebra $\mathscr{H}$ is generated by a single function $\psi\left(x_{1}, x_{2}\right)=x_{1}^{3}+x_{2}$, which means that the Hermann-Krener condition does not hold at any point. The discretized system $\Sigma_{\mathbb{T}}$ is given by

$$
\begin{gathered}
x_{1}\left(t+\mu_{\mathbb{T}}(t)\right)=x_{1}(t)-\mu_{\mathbb{T}}(t) u(t), \\
x_{2}\left(t+\mu_{\mathbb{T}}(t)\right)=x_{2}(t)+3 \mu_{\mathbb{T}}(t) x_{1}^{2}(t) u(t), \\
y(t)=x_{1}(t)^{3}+x_{2}(t) .
\end{gathered}
$$

The observation algebra $\mathscr{H}_{\mathbb{T}}$ contains now the functions $\psi_{1}\left(x_{1}, x_{2}\right)=x_{1}^{3}+x_{2}$ and $\psi_{2}\left(x_{1}, x_{2}\right)=x_{1}$. The HermannKrener condition is then satisfied at all points and for all discrete time-scales $\mathbb{T}$.

Remark 31. Hermann-Krener rank condition is equivalent to the property that the ideal $J_{x_{0}}$ is maximal; that is, it is generated by the coordinate functions. One can show that this property is preserved when the ideal $J_{x_{0}}$ is replaced with the ideals corresponding to systems $\Sigma_{\mathbb{T}}$ if $\mathbb{T}$ contains $t$ with $\mu_{\mathbb{T}}(t)$ sufficiently small. In characterizations of weak and robust local observability there appear real radicals of ideals. It is not clear whether desired properties of the radicals like maximality (for $\operatorname{WLO}\left(x_{0}\right)$ ) or nonproperness (for $\operatorname{RLO}\left(x_{0}\right)$ are preserved under discretizations. Thus preservation of weak and robust local observability under Euler discretization is still an open problem.

We finish this discussion with a positive example.

Example 32. Let $\Sigma$ be

$$
\dot{x}_{1}=x_{2} u, \quad \dot{x}_{2}=-x_{1} u, \quad y=x_{1}^{2}+x_{2}^{2} .
$$

The observation algebra of $\Sigma$ is generated by $\varphi\left(x_{1}, x_{2}\right)=x_{1}^{2}+$ $x_{2}^{2}$.

The discretization gives $\Sigma_{\mathbb{T}}$

$$
x_{1}^{\Delta}=x_{2} u, \quad x_{2}^{\Delta}=-x_{1} u, \quad y=x_{1}^{2}+x_{2}^{2},
$$

where

$$
x^{\Delta}(t)=\frac{x\left(t+\mu_{\mathbb{T}}(t)\right)-x(t)}{\mu_{\mathbb{T}}(t)} .
$$

The observation algebra of $\Sigma_{\mathbb{T}}$ is also generated by $\varphi\left(x_{1}, x_{2}\right)=$ $x_{1}^{2}+x_{2}^{2}$.

Thus, $\Sigma$ and $\Sigma_{\mathbb{T}}$ are both weakly locally observable at $x=$ 0 . They are not weakly locally observable at any other point.

Example 27 describes a positive behavior of robust local observability under discretization. In fact, the calculations are the same for all time-scales.

\section{Conclusions}

We have shown that the methods of real analytic geometry and real algebra developed for continuous time systems may be used for systems on arbitrary time-scales, in particular on the scale of integers and on the quantum scales. Different concepts of local observability for systems on arbitrary timescales have been considered. We have established relations between these concepts and provided characterizations of weak and robust local observability with the aid of certain ideals of the ring of germs of analytic functions and real radicals of those ideals. Equivalent geometric characterizations have been given. Observation algebras from which the ideals are obtained and the ideals themselves depend on the time-scale on which the systems is defined, but once the ideals are computed, the procedures and the criteria of local observability are the same for all time-scales. This allows for unified treatment of observability of systems on arbitrary time-scales.

The language of time-scales allows for a natural description of discretization of continuous-time systems: the ordinary derivative is replaced by delta derivative on a discrete time-scale $\mathbb{T}$. The paper contains preliminary results on preservation of properties related to observability under discretization. In particular Hermann-Krener rank condition is preserved. Preservation of other properties, in particular weak and robust local observability, is stated as an open problem. To solve the problem one will have to study limit properties of real radicals for rings of germs of analytic functions. This will be a subject of a future research.

\section{Appendices}

\section{A. Calculus on Time-scales}

A time-scale $\mathbb{T}$ is an arbitrary nonempty closed subset of the set $\mathbb{R}$ of real numbers. In particular $\mathbb{R}, h \mathbb{Z}$ for $h>0$ and $q^{\mathbb{N}}:=$ $\left\{q^{k}, k \in \mathbb{N}\right\}$ for $q>1$ are time-scales. We assume that $\mathbb{T}$ is a topological space with the relative topology induced from $\mathbb{R}$. If $t_{0}, t_{1} \in \mathbb{T}$, then $\left[t_{0}, t_{1}\right]_{\mathbb{T}}$ denotes the intersection of the ordinary closed interval with $\mathbb{T}$. Similar notation is used for open, half-open or infinite intervals.

For $t \in \mathbb{W}$ we define the forward jump operator $\sigma: \mathbb{T} \rightarrow \mathbb{\mathbb { T }}$ by $\sigma(t):=\inf \{s \in \mathbb{T}: s>t\}$ if $t \neq \sup \mathbb{\mathbb { V }}$ and $\sigma(\sup \mathbb{\mathbb { T }})=\sup \mathbb{\mathbb { V }}$ when sup $\mathbb{T}$ is finite; the backward jump operator $\rho: \mathbb{T} \rightarrow \mathbb{T}$ by $\rho(t):=\sup \{s \in \mathbb{T}: s<t\}$ if $t \neq \inf \mathbb{T}$ and $\rho(\inf \mathbb{T})=\inf \mathbb{T}$ when inf $\mathbb{T}$ is finite; the forward graininess function $\mu: \mathbb{T} \rightarrow$ $[0, \infty)$ by $\mu(t):=\sigma(t)-t$; the backward graininess function $\nu: \mathbb{T} \rightarrow[0, \infty)$ by $\nu(t):=t-\rho(t)$.

If $\sigma(t)>t$, then $t$ is called right-scattered, while if $\rho(t)<t$, it is called left-scattered. If $t<\sup \mathbb{\mathbb { V }}$ and $\sigma(t)=t$, then $t$ is called right-dense. If $t>\inf \mathbb{T}$ and $\rho(t)=t$, then $t$ is leftdense.

The time-scale $\mathbb{T}$ is homogeneous, if $\mu$ and $\nu$ are constant functions. When $\mu \equiv 0$ and $\nu \equiv 0$, then $\mathbb{T}=\mathbb{R}$ or $\mathbb{T}$ is a closed interval (in particular a half-line). When $\mu$ is constant and greater than 0 , then $\mathbb{T}=\mu \mathbb{Z}+c$, for some $c \in \mathbb{R}$.

Let $\mathbb{T}^{\kappa}:=\{t \in \mathbb{T}: t$ is nonmaximal or left-dense $\}$. Thus $\mathbb{T}^{\mathcal{k}}$ is obtained from $\mathbb{T}$ by removing its maximal point if this point exists and is left-scattered.

Let $f: \mathbb{T} \rightarrow \mathbb{R}$ and $t \in \mathbb{T}^{\mathcal{K}}$. The delta derivative of $f$ at $t$, denoted by $f^{\Delta}(t)$, is the real number with the property that 
given any $\varepsilon$ there is a neighborhood $U=(t-\delta, t+\delta)_{\mathbb{W}}$ such that

$$
\left|(f(\sigma(t))-f(s))-f^{\Delta}(t)(\sigma(t)-s)\right| \leq \varepsilon|\sigma(t)-s|
$$

for all $s \in U$. If $f^{\Delta}(t)$ exists, then we say that $f$ is delta differentiable at $t$. Moreover, we say that $f$ is delta differentiable on $\mathbb{T}^{k}$ provided $f^{\Delta}(t)$ exists for all $t \in \mathbb{T}^{k}$.

Example A.1. If $\mathbb{T}=\mathbb{R}$, then $f^{\Delta}(t)=f^{\prime}(t)$. If $\mathbb{T}=h \mathbb{Z}$, then $f^{\Delta}(t)=(f(t+h)-f(t)) / h$. If $\mathbb{T}=q^{\mathbb{N}}$, then $f^{\Delta}(t)=(f(q t)-$ $f(t)) /((q-1) t)$.

A function $f: \mathbb{T} \rightarrow \mathbb{R}$ is called $r d$-continuous provided it is continuous at right-dense points in $\mathbb{T}$ and its left-sided limits exist (finite) at left-dense points in $\mathbb{T}$. If $f$ is continuous, then it is rd-continuous.

A function $f: \mathbb{T} \rightarrow \mathbb{R}$ is called regressive, if $1+$ $\mu(t) f(t) \neq 0$ for all $t \in \mathbb{T}$.

A function $F: \mathbb{T} \rightarrow \mathbb{R}$ is called an antiderivative of $f: \mathbb{T} \rightarrow \mathbb{R}$ provided $F^{\Delta}(t)=f(t)$ holds for all $t \in \mathbb{T}^{\kappa}$. Let $a, b \in \mathbb{T}$. Then the delta integral of $f$ on the interval $[a, b)_{\mathbb{T}}$ is defined by

$$
\int_{a}^{b} f(\tau) \Delta \tau:=\int_{[a, b)_{\top}} f(\tau) \Delta \tau:=F(b)-F(a) .
$$

Riemann and Lebesgue delta integrals on time-scales have been also defined (see, e.g., [13]). It can be shown that every rd-continuous function has an antiderivative and its Riemann and Lebesgue integrals agree with the delta integral defined above.

Example A.2. If $\mathbb{T}=\mathbb{R}$, then $\int_{a}^{b} f(\tau) \Delta \tau=\int_{a}^{b} f(\tau) d \tau$, where the integral on the right is the usual Riemann integral. If $\mathbb{T}=$ $h \mathbb{Z}, h>0$, then $\int_{a}^{b} f(\tau) \Delta \tau=\sum_{t=(a / h)}^{(b / h)-1} f(t h) h$ for $a<b$.

\section{B. Basic Real Geometry}

We assume that the reader is familiar with the concepts of germs of functions and sets, and with fundamentals of the sheaf theory and theory of analytic sets. Necessary definitions can be found, for example, in [14,15]. If $B$ is a "global" object (a set, a function, or a family of functions), $B_{x}$ will always denote its germ at the point $x$ (but the precise meaning of the germ will depend on the meaning of the object). If $C$ is a germ, $\widetilde{C}$ will denote one of its representatives. By $\mathcal{O}_{x}$ we denote the algebra of germs of real analytic functions at $x$, where $x \in \mathbb{R}^{n}$ ( $n$ fixed throughout the paper), and by $m_{x}$ the (only) maximal ideal of $\mathcal{O}_{x}$, consisting of all germs in $\mathcal{O}_{x}$ that vanish at $x$. By $\mathcal{O}$ we denote the sheaf of germs of real analytic functions on $\mathbb{R}^{n}$.

If $U$ is an open subset in $\mathbb{R}^{n}$, then $\mathcal{O}_{U}$ will mean the algebra of real analytic functions on $U$. If $A$ is a subalgebra of $\mathcal{O}_{U}$ and $x_{0} \in U$, then $A_{x_{0}}$ means the set of germs at $x_{0}$ of functions from $A$. Of course $A_{x_{0}}$ is again an algebra over $\mathbb{R}$. If $I$ is an ideal of $\mathcal{O}_{U}$, then $I_{x_{0}}$ means the ideal of $\mathcal{O}_{x_{0}}$ generated by the germs at $x_{0}$ of function from $I$.

Consider a set-germ $A$ in $\mathbb{R}^{n}$ (at some point $x$ ). Then $J(A)$ denotes the ideal of $\mathcal{O}_{x}$ consisting of germs (at $x$ ) of real analytic functions that vanish on $A$. If $I$ is an ideal of $\mathcal{O}_{x}$, then $Z(I)$ will denote the zero set-germ of $I$ (at $x)$. Let us recall that $Z(I)$ is defined as the intersection of the set-germs $Z\left(\varphi_{i}\right)$, $i=1, \ldots, k$, where $\varphi_{1}, \ldots, \varphi_{k}$ are generators of the ideal $I$. Since only finite intersections of set-germs are defined, we must use here the property that $\mathcal{O}_{x}$ is Noetherian.

We have a natural duality between ideals and set-germs. If $I_{1} \subset I_{2}$, then $Z\left(I_{2}\right) \subset Z\left(I_{1}\right)$.

Let $P$ be any commutative ring with a unit and let $I$ be an ideal of $P$. Then the real radical of $I$, denoted by $\sqrt[R]{I}$, is the set of all $a \in P$ for which there is $m \in \mathbb{N}, k \in \mathbb{N} \cup\{0\}$ and $b_{1}, \ldots, b_{k} \in P$ such that

$$
a^{2 m}+b_{1}^{2}+\cdots+b_{k}^{2} \in I .
$$

The real radical is an ideal in $P$ and it contains $I$. If $I$ is a proper ideal of $P$, then also $\sqrt[\mathbb{R}]{I}$ is proper. An ideal $I$ is called real if $\sqrt[R]{I}=I$

Theorem B.1 (see [16]). Let $x \in \mathbb{R}^{n}$. If I is an ideal of $\mathcal{O}_{x}$, then

$$
J(Z(I))=\sqrt[\mathbb{R}]{I}
$$

Theorem B.1 implies that there is a 1:1 correspondence between germs of analytic sets at $x$ and real ideals of $\mathcal{O}_{x}$.

\section{Acknowledgment}

This work was supported by the Bialystok University of Technology Grant S/WI/2/2011.

\section{References}

[1] R. Hermann and A. J. Krener, "Nonlinear controllability and observability," IEEE Transactions on Automatic Control, vol. 22, no. 5, pp. 728-740, 1977.

[2] Z. Bartosiewicz, "Local observability of nonlinear systems," Systems \& Control Letters, vol. 25, no. 4, pp. 295-298, 1995.

[3] Z. Bartosiewicz, "Real analytic geometry and local observability," in Differential Geometry and Control (Boulder, CO, 1997), G. Ferreyra, R. Gardner, H. Hermes, and H. Sussmann, Eds., vol. 64 of Proc. Sympos. Pure Math., pp. 65-72, American Mathematical Society, Providence, RI, USA, 1999.

[4] F. Albertini and D. D’Alessandro, "Observability and forwardbackward observability of discrete-time nonlinear systems," Mathematics of Control, Signals, and Systems, vol. 15, no. 4, pp. 275-290, 2002.

[5] D. Mozyrska and Z. Bartosiewicz, "Algebraic criteria for stable local ob- servability of analytic systems on $\mathbb{R}^{n}$," in Proceedings of European Control Conference (ECC '97), Brussels, Belgium, July 1997.

[6] Z. Bartosiewicz, "Observability of linear positive systems on time scales," in Proceedings of the 51st IEEE Conference on Decision and Control, pp. 2581-2586, Maui, Hawaii, December 2012.

[7] Z. Bartosiewicz and E. Pawluszewicz, "Realizations of nonlinear control systems on time scales," IEEE Transactions on Automatic Control, vol. 53, no. 2, pp. 571-575, 2008.

[8] E. Pawłuszewicz, "Observability of nonlinear control systems on time scales," International Journal of Systems Science, vol. 43, no. 12, pp. 2268-2274, 2012. 
[9] H. J. Sussmann, "Existence and uniqueness of minimal realizations of nonlinear systems," Mathematical Systems Theory, vol. 10, no. 3, pp. 263-284, 1976/77.

[10] Z. Bartosiewicz and J. Johnson, "Systems on universe spaces," Acta Applicandae Mathematicae, vol. 37, no. 3, pp. 233-266, 1994.

[11] R. Narasimhan, Introduction to the Theory of Analytic Spaces, Lecture Notes in Mathematics, no. 25, Springer, Berlin, Germany, 1966.

[12] H. Cartan, "Variétés analytiques réelles et variétés analytiques complexes," Bulletin de la Société Mathématique de France, vol. 85, pp. 77-99, 1957.

[13] G. Sh. Guseinov, "Integration on time scales," Journal of Mathematical Analysis and Applications, vol. 285, no. 1, pp. 107-127, 2003.

[14] R. C. Gunning and H. Rossi, Analytic Functions of Several Complex Variables, Prentice-Hall, Englewood Cliffs, NJ, USA, 1965.

[15] F. Guaraldo, P. Macrì, and A. Tancredi, Topics on Real Analytic Spaces, Advanced Lectures in Mathematics, Friedr. Vieweg \& Sohn, Braunschweig, Germany, 1986.

[16] J.-J. Risler, "Le théorème des zéros en géométries algébrique et analytique réelles," Bulletin de la Société Mathématique de France, vol. 104, no. 2, pp. 113-127, 1976. 


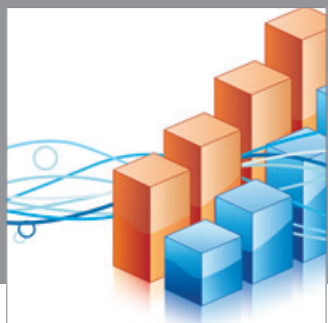

Advances in

Operations Research

mansans

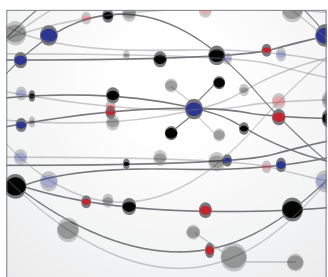

The Scientific World Journal
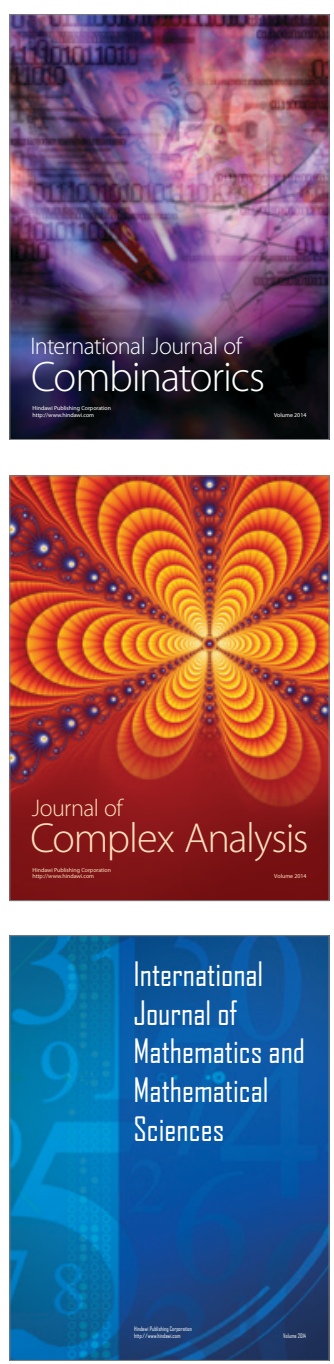
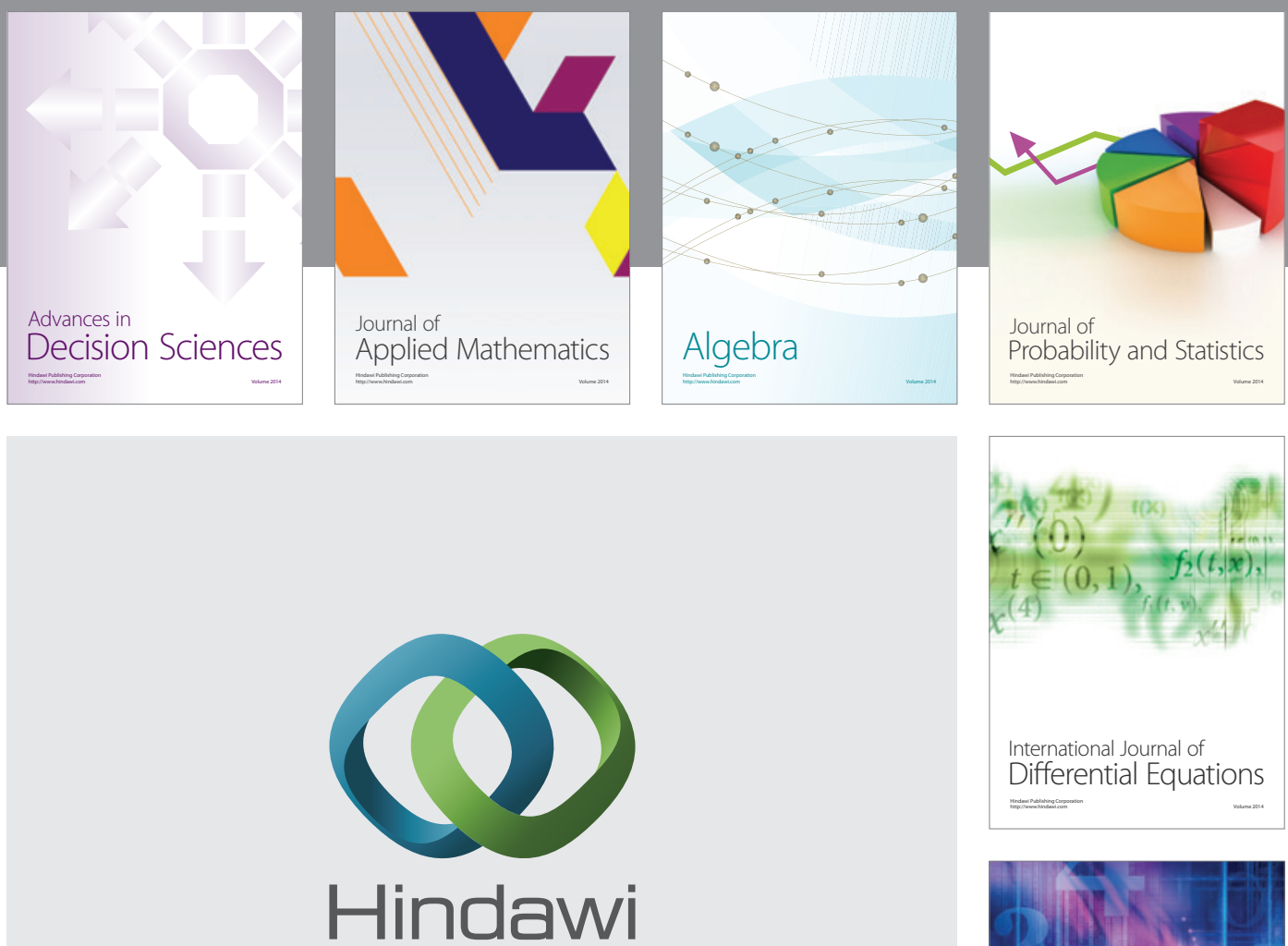

Submit your manuscripts at http://www.hindawi.com
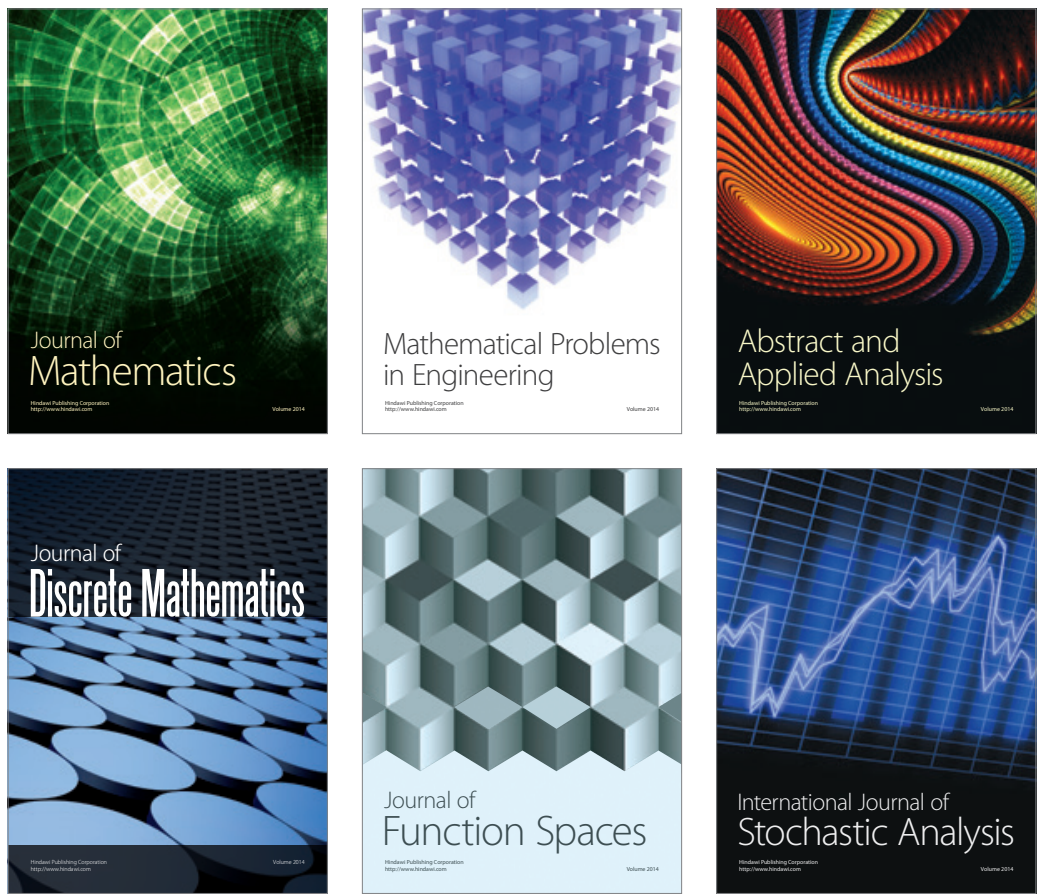

Journal of

Function Spaces

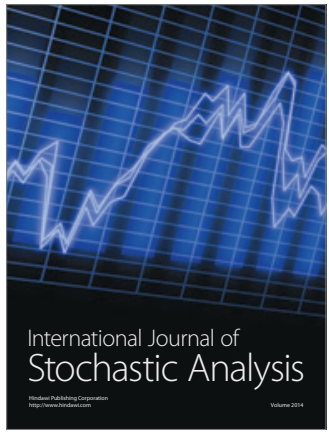

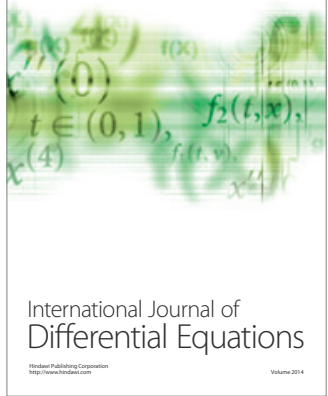
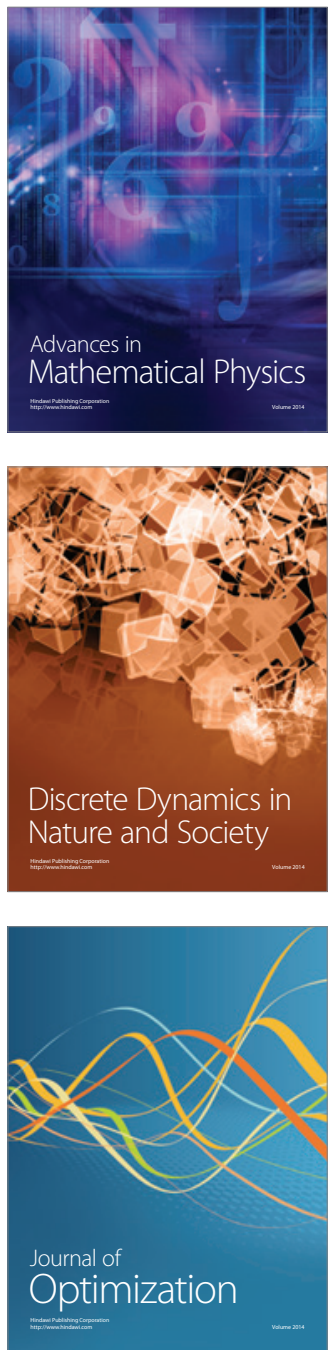